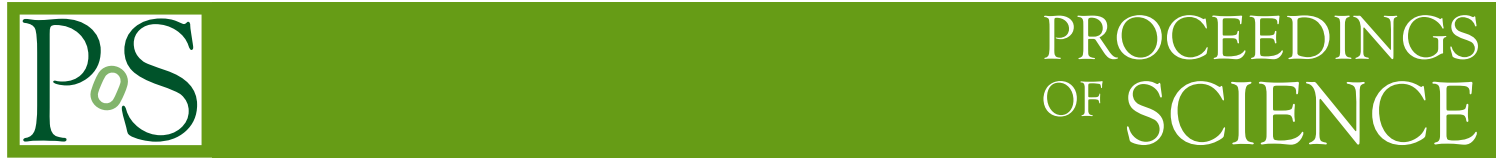

\title{
The one-flavor quark condensate and related problems
}

\section{Thomas DeGrand*}

Department of Physics, University of Colorado, Boulder CO 80309 USA

E-mail: degrandepizero.colorado.edu

\begin{abstract}
I describe a recent calculation (by me, Hoffmann, Liu, and Schaefer) of the chiral condensate in one-flavor QCD using numerical simulations with overlap fermions. The condensate is extracted by fitting the distribution of low lying eigenmodes of the Dirac operator in sectors of fixed topological charge to the predictions of Random Matrix Theory. Our results are in excellent agreement with estimates from the orientifold large-N expansion. Much interesting physics surrounds this calculation, which I will highlight.
\end{abstract}

XXIVth International Symposium on Lattice Field Theory

July 23-28, 2006

Tucson, Arizona, USA

${ }^{*}$ Speaker. 
Theories like QCD, but with small differences, can teach us things about QCD. QCD with one flavor of dynamical fermion is such a theory: it is related, by a remarkable path, to the $N_{c} \rightarrow \infty$ limit of $\mathscr{N}=1$ supersymmetric Yang-Mills theory, especially to one of its exactly-known observables, the gluino condensate.

We lattice people are not the only ones interested in strongly coupled gauge fi eld theories. Supersymmetry is a powerful tool for understanding these systems, and it is an important and active area of research, to extend results from supersymmetric theories to non-supersymmetric ones. One way to do this is to replace the degrees of freedom in supersymmetric fi eld theories with new ones, while still preserving desirable features. The large- $N_{c}$ (number of colors) limit is an important part of this program. (For an early attempt, see [1].)

Recently, Armoni, Shifman, and Veneziano $[2,3,4,5]$ (ASV) suggested a new large- $N_{c}$ expansion with some remarkable features. In contrast to the 't Hooft large- $N_{c}$ limit [6] $\left(N_{c} \rightarrow \infty, g^{2} N_{c}\right.$ and $N_{f}$ fi xed, with quarks in the fundamental representation of $S U\left(N_{c}\right)$ ), quarks are placed in the two-index antisymmetric representation of $S U\left(N_{c}\right)$. Now in the $N_{c} \rightarrow \infty, g^{2} N_{c}$ and $N_{f}$ fi xed limit of QCD, quark effects are not decoupled, because there are as many quark degrees of freedom as gluonic ones, $O\left(N_{c}^{2}\right)$ in either case. In Ref. [5] the authors have argued that a bosonic sector of $\mathscr{N}=1$ super-Yang-Mills (SYM) theory is equivalent to this theory in the large- $N_{c}$ limit. $\mathscr{N}=1 \mathrm{SYM}$ is a theory of adjoint gluons and their gluino (Majorana fermion) partners, and the equivalence of these theories in perturbation theory can be seen by comparing the vertices, as in Fig. 1, taken from Ref. [7]. The large- $N_{c}$ QCD-like theory is called "orientifold QCD."

The perturbative connection of orientifold QCD to $\mathscr{N}=1 \mathrm{SYM}$ is uncontroversial. In Ref. [5], ASV have presented a nonperturbative proof of the connection. This proof has been extended by Patella [8] to lattice regularized theories. Recently, Yaffe and Ünsal [9] have argued that the proof of ASV is incomplete: that orientifold QCD and $\mathscr{N}=1 \mathrm{SYM}$ have a different phase structure on spacetimes with small compact dimensions, in which charge conjugation symmetry is spontaneously broken. Only when the two theories have identical vacua can the proof hold.

I am certainly not competent to comment more on this subject, so let us see what orientifold QCD might have to do with a lattice project: For $N_{c}=3$, orientifold QCD is equivalent to QCD with a single quark flavor in the fundamental representation of $S U(3)$. This equivalence can be seen in the first and second terms in the $\beta$ function and in the lowest order anomalous dimension for the running quark mass (or quark condensate), as Table 1, taken from Ref. [7] shows. This means that if the proof of nonperturbative equivalence is correct, nonperturbative quantities (in the bosonic sector) computed in super-Yang-Mills theory can be related to corresponding ones in one-flavor QCD, up to $1 / N_{c}$ effects.

The analog of the quark condensate in ordinary QCD is the gluino condensate in $\mathscr{N}=1 \mathrm{SYM}$. It can be calculated exactly in large- $N_{c}$ using saddle point methods (Ref. [10] is a recent reference with a complete citation path). ASV used this exact result to estimate [4] the quark condensate in one-flavor QCD from the value of the gluino condensate in SYM. They found (with our sign conventions)

$$
\Sigma=\{0.014,0.021,0.028\} \mathrm{GeV}^{3}
$$

in the $\overline{M S}$ scheme at $\mu=2 \mathrm{GeV}$. The spread of values gives their estimate of $1 / N_{c}$ corrections (basically $1 \pm 1 / 3$ ). 


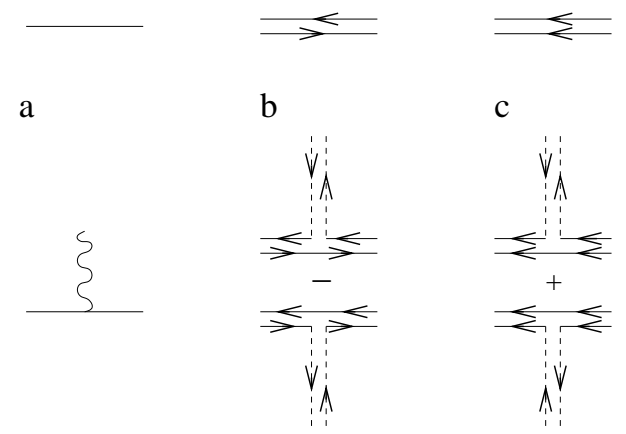

Figure 1: (a) The fermion propagator and the fermion-fermion-gluon vertex. (b) $\mathscr{N}=1$ SYM theory. (c) Orientifold daughter

Now we come to the lattice: Last November, Schaefer and I had most of the solution to the problem of how to do Hybrid Monte Carlo for any $N_{f}$, using overlap fermions[11]. But what to do with it? I remembered the ASV prediction. Over Christmas vacation we started simulations to check this number and the preprint [12] came out in May. We hit their number bang on!

We have to deal with a certain amount of imprecise language related to the condensate: The quantity $\langle\bar{q} q\rangle$ is one defi nition of the condensate. Rather than measuring it directly, we will determine the particular combination of the coeffi cients of the low energy effective fi eld theory, $\Sigma=f B$, in the usual parameterization for $N_{f}>1 \mathrm{QCD}$,

$$
\mathscr{L}_{2}=\frac{f^{2}}{4} \operatorname{Tr}\left(\partial_{\mu} U \partial_{\mu} U^{\dagger}\right)+B \frac{f^{2}}{2} \operatorname{Tr}\left[M\left(U+U^{\dagger}\right)\right]
$$

One expects that the quantity $\langle\bar{q} q\rangle$ (as computed, for example, in a lattice simulation at some quark mass $m_{q}$ and simulation volume $V$ ) is a function of $\Sigma, f, m_{q}$, and simulation volume $V$. $\Sigma$ and $f$ are the interesting quantities, and a direct lattice measurement of $\langle\bar{q} q\rangle$ from several quark masses would have to be converted to a prediction of $\Sigma$ and $f$, by fi tting it to the appropriate functional form from chiral perturbation theory. The same thing would have to be done if one measured observables, like the pseudoscalar mass and $f_{\pi}$, and used the GMOR relation to infer $\Sigma$.

$N_{f}=1 \mathrm{QCD}$ is a peculiar theory. Chiral symmetry is anomalous. There are no Goldstone bosons, just the eta-prime, which gets its mass through the anomaly. The $\Sigma$ which we are about to extract is therefore not an order parameter of spontaneous chiral symmetry breaking. However, there still exists a well defi ned low-energy description of $N_{f}=1$ QCD. It has been given by Leutwyler and Smilga [13] to which we refer the reader for details. They show that up to terms of order $m^{2} V$ the partition function is

$$
Z=\exp \left\{\Sigma V \operatorname{Re}\left(m e^{-i \theta}\right)\right\}
$$

with $\theta$ the vacuum angle. $\Sigma$ is the infi nite volume zero quark mass limit of $-\left\langle{ }^{-} q q\right\rangle$ at $\theta=0$.

There is a reasonably inexpensive way to directly compute $\Sigma$ : it involves measuring the distribution of the low eigenmodes of the Dirac operator, in sectors of fi xed topology $v$ in a simulation volume $V$. The probability distribution of individual eigenvalues $\lambda_{n}$ is given by Random Matrix 


\begin{tabular}{|c|c|c|c|}
\hline$\frac{\text { Theory } \rightarrow}{\text { Coeff } \downarrow}$ & $1 \mathrm{f}-\mathrm{QCD}$ & Orienti A & S YM \\
\hline \hline$\beta_{0}$ & $\frac{11}{3} N-\frac{2}{3}$ & $3 N+\frac{4}{3}$ & $3 N$ \\
\hline$\beta_{1}$ & $\frac{17}{3} N^{2}-\frac{13}{6} N+\frac{1}{2 N}$ & $3 N^{2}+\frac{19}{3} N-\frac{4}{N}$ & $3 N^{2}$ \\
\hline$\gamma$ & $\frac{3\left(N^{2}-1\right)}{2 N}$ & $\frac{3(N-2)(N+1)}{N}$ & $3 N$ \\
\hline
\end{tabular}

Table 1: A comparison of the beta functions and anomalous dimensions for the three theories

Theory (RMT) $[14,15,16]$ as a function of the dimensionless quantity $\lambda_{n} \Sigma V$, which depends parametrically on the combination $m_{q} \Sigma V$ and, of course, $N_{f}$. We use the specifi c method and predictions from Refs. $[17,18]$.

The connection is only supposed to work deep in the $\varepsilon$ regime of QCD, but the eigenmode distribution is very robust and this does not seem to be a necessary constraint in practice.

So the calculation involves several parts:

- Perform simulations in sectors of fi xed topology

- Measure the eigenmodes and fit their distributions to the RMT expression: this gives the lattice-regulated $a^{3} \Sigma$

- Determine $a$ : we used the potential and the Sommer parameter [19]

- Convert to $\overline{M S}$ : we used the RI-MOM method [20]

How we did the simulations [11] is worth a paragraph. The last two items are pretty straightforward. So is computing the eigenmodes. Fitting the distributions is also a little nonstandard, so I'll fil that in, too.

When one is dealing with chiral symmetry on the lattice, it is very convenient to work with a fermion action which is chiral. That way, the physics of spontaneous symmetry breaking (including the anomaly) is not masked by explicit chiral symmetry breaking from the lattice discretization. While standard lore says that it is in principle possible to correct for chiral symmetry breaking effects in the bare action during the analysis, in practice this can be diffi cult and there is always the danger of new effects which one did not plan on (like exceptional confi gurations for Wilson-type actions). Why go looking for trouble?

Thus we are led to use overlap [21, 22] fermions, which exactly encode chiral symmetry through the Ginsparg-Wilson [23] relation. It happens that, as an extra treat, it is possible to simulate any number of flavors of overlap fermions, without requiring any degeneracy in the quark mass spectrum, using the exact Hybrid Monte Carlo algorithm.

Due to the Ginsparg-Wilson relation, overlap fermions have the nice properties that the spectrum of the squared massless Dirac operator $H^{2}=D^{\dagger} D$ commutes with $\gamma_{5}$ and has degenerate opposite-chirality eigenfunctions, apart from chiral zero modes and their partners at $H^{2}=4 R_{0}^{2}$ (where $R_{0}$ is the radius of the Ginsparg-Wilson circle). The corresponding eigenmodes of $H=\gamma_{5} D$ and $D$ itself can be found by diagonalizing the $2 \times 2$ degenerate subspaces of $H^{2}$. Then $[24,25]$ the 
contribution of the paired modes to the determinant of a single flavor of overlap fermions is given by $\operatorname{det} H_{\sigma}^{2}$, the determinant of $H^{2}$ evaluated in a single chirality sector. It is included in the action with a single chiral pseudofermion for each flavor, $\Delta S=\phi_{\sigma} H_{\sigma}(m)^{-2} \phi_{\sigma}$. The contribution of the zero modes can be included in the HMC by direct addition to the action at a topological boundary (the extra factor is $N_{f}|Q| \ln \left(m_{q} /\left(2 R_{0}\right)\right)$ for $N_{f}$ degenerate flavors). For analysis, there is a different weighting of $v=0$ and $v \neq 0$ confi gurations.

What was never written down (maybe it is trivial) is how to initialize the pseudofermions: we need to generate random numbers to initialize $\phi_{\sigma}$. The trick is to begin with a chiral random source $R_{\sigma}$ and to use the Zolotarev formula to construct $\phi_{\sigma}=\sqrt{H_{\sigma}(m)^{2}} R_{\sigma}$.

We used the algorithm of Ref. [26]. In it, one must monitor the eigenmodes of the "kernel operator" $h\left(-R_{0}\right)$ in $D=R_{0}\left(1+\gamma_{5} \varepsilon(h(-R 0))\right)$. When it develops a zero mode, the topology of the underlying gauge confi guration changes and with it, there is a step discontinuity in the fermionic action. Random matrix theory wants eigenmodes in sectors of fi xed topology. We generate those simply by forbidding tunneling events in the molecular dynamics evolution, and evolving in sectors of fi xed $v$.

To do the calculation, we collected data on a few P4's and P4E's for a few months, on $10^{4}$ and $8^{3} \times 12$ lattices, at a lattice spacing of about $0.15 \mathrm{fm}$.. (The $10^{4}$ data set had about 500 trajectories each for winding number $v=0$ and 1.)

Dynamical overlap is not cheap but it is completely feasible for small projects as long as one is willing to be creative (or maybe Baroque?) [27, 28]. The essential ingredient is a fat link gauge connection. We used stout links [29], three steps with $\rho=0.15$. As far as the overlap goes, more smearing is better and 3 times 0.15 is about 2.5 times faster than the 2 times 0.15 of our previous work [27, 28]. The improvement comes from decoupling the fermions from UV gauge fluctuations which would generate small eigenmodes of $h\left(-R_{0}\right)$. This decreases the condition number of $h\left(-R_{0}\right)$ and speeds up the calculation of $D$. The dark side (if there is one) of a fat link action is that the fat links make the action more spread out than a conventional thin link action. Minimizing this spread is part of "action engineering," like minimizing the range of the fermionic couplings. We have performed the usual tests of locality on our action and never seen anything peculiar. Remember, thin links and fat links are just choices for the bare action which differ (in a Symanzik sense) through irrelevant operators. Formally, thin and fat link actions are both in the universality class of QCD. We are allowed to tune irrelevant operators as we please to ease the computational burden while preserving symmetries - not to do so is bad software engineering.

If one wants to use eigenmodes, a kernel which looks "overlap-like" is also essential, otherwise the eigenmode part of the code is prohibitively expensive. (I have never been able to do anything with thin link Wilson-kernel overlap; it is too expensive for work stations. The problem is in the eigensolver: one begins with a set of trial eigenmodes which are then iteratively improved. Without some good idea, the beginning modes are typically random vectors. Improving them takes many iterations, which when done with the overlap action is very slow. One can gain a lot of time using better eigenmodes, from some "overlap-like" action, as an intermediate step. The overlap actions I use are built on "overlap-like" kernels, but the overlap action with a Wilson kernel has a very different spectrum from a Wilson action.)

All of this is well documented for quenched simulations [30]. I can't help thinking that there are more tricks out there. Oh, for another factor of fi ve speedup... 
So we collected a set of fermionic eigenmodes. We want to fi t their probability distributions to RMT formulas. The analysis is a little nonstandard, because the eigenmodes are continuously distributed. A referee led us to the Kolmogorov-Smirnov test [31] as a measure for the goodness of the fi t. It compares the cumulative distribution function of the data $C(x)$ to the theoretical prediction $P(x)=\int_{-\infty}^{x} f(x) \mathrm{d} x . C(x)$ is the fraction of eigenvalues with a value smaller than $x$.

The quantity of interest is the largest deviation of $P$ and $C: D=\max _{x}|P(x)-C(x)|$. From this the confi dence level is given by

$$
Q_{K S}((\sqrt{N}+0.12+0.11 / \sqrt{N}) D)
$$

with

$$
Q_{K S}(\lambda)=2 \sum_{j=1}^{\infty}(-)^{j-1} \exp \left(-2 j^{2} \lambda^{2}\right)
$$

In fi ts to a single eigenmode distribution we maximize this quantity. When fi tting to more than one mode, we maximize the product over the individual confi dence levels. The errors on the fit parameter $\Sigma$ are determined by the bootstrap procedure. An example of such a fit is shown in Fig. 2. After a lot of angst (which modes to fi t, what about correlations...) $\Sigma a^{3}$ turned out to be remarkably robust: it didn't matter what we did.

Completing the calculation with the lattice spacing from the Sommer parameter and the matching factor from RI-MOM, we found

$$
\begin{aligned}
r_{0}^{3} \Sigma(\overline{M S}, \mu=2 \mathrm{GeV}) & =Z_{s}(\mu, a) \times \Sigma a^{3} \times\left(\frac{r_{0}}{a}\right)^{3} \\
& =(0.86(3)) \times(0.0096(3)) \times(3.37(10))^{3} \\
& =0.317(22) .
\end{aligned}
$$

With $r_{0}=0.5 \mathrm{fm}$, this is

$$
\Sigma(\overline{M S}, \mu=2 \mathrm{GeV})=0.0194(20) \mathrm{GeV}^{3}
$$

which agrees pretty nicely with Eq. 1 .

The summary of McNeile [32] shows that the $N_{c}=3$ condensate is not very $N_{f}$ dependent. Indeed, Schaefer, Liu and I just fi nished [33] an $N_{f}=2$ measurement using basically identical techniques to what I have described here, and we fi nd $\Sigma=0.0225(25) \mathrm{GeV}^{3}=(282(10) \mathrm{MeV})^{3}$. There is actually an annoying systematic in this number: In fi nite volume, there is a fi rst order correction to the condensate, basically the one loop graph from Goldstone bosons which are emitted from the propagating pseudoscalar and which are absorbed at an image point of the vertex. The correction is $\Sigma \rightarrow \rho \Sigma$, where

$$
\rho=1+\frac{N_{f}^{2}-1}{N_{f}} \frac{c\left(l_{i} / l\right)}{f_{\pi}^{2} L^{2}}
$$

and $c\left(l_{i} / l\right)$ depends on the geometry[34]. This term is absent in $N_{f}=1$ because there are no Goldstones. We haven't measured $f_{\pi}$, but with $93 \mathrm{MeV}$, in our volume for $N_{f}=2, \rho \sim 1.43$. Fortunately, people publish $\Sigma^{1 / 3}$, not $\Sigma$ !

After the paper came out, Veneziano reminded me that their prediction was really for $\Sigma / \Lambda^{3}$ "without going through actual experimental numbers." For us lattice people, a ratio of nonperturbative quantities with no (or minimal) intrusion of perturbation theory is much cleaner than $\Sigma / \Lambda^{3}$, 


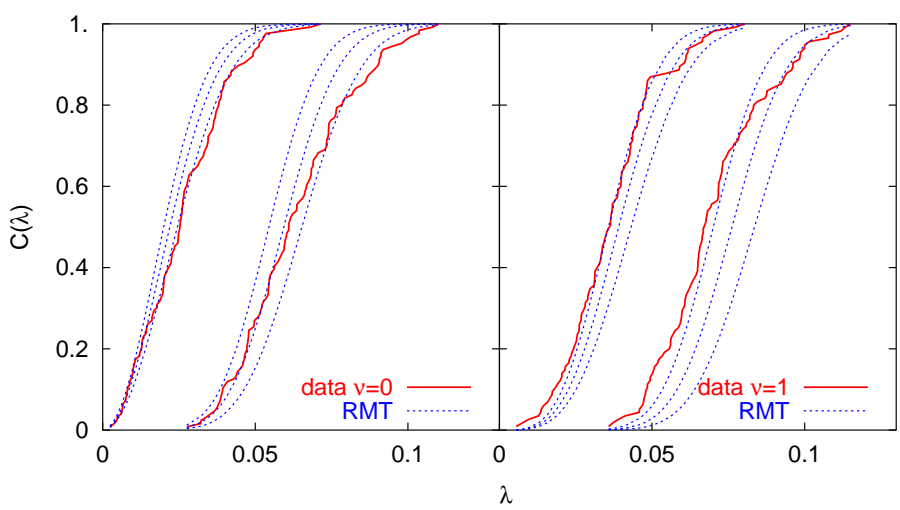

Figure 2: Cumulative distributions from RMT fit to eigenmode distributions. For each mode, the three dotted lines, running from left to right, correspond to fits to the lowest mode in the $v=1$ sector only, the lowest mode $v=0$ and $|v|=1$, and the lowest mode in $v=0$ only.

and the defi nition of $\Lambda$ is exquisitely sensitive to a determination of a coupling: recall that

$$
a \Lambda=\left(\frac{16 \pi^{2}}{\beta_{0} g^{2}(a)}\right)^{\beta_{1} /\left(2 \beta_{0}^{2}\right)} \exp \left(-\frac{8 \pi^{2}}{\beta_{0} g^{2}(a)}\right) .
$$

Tadpole-ology (plaquette $\rightarrow \alpha_{V}\left(q^{*}\right) \rightarrow \Lambda$ ) at one value of the lattice spacing is just too unstable to be useful. However, the ALPHA collaboration has used the Schrödinger functional real space renormalization group to compute $[35,36]$ the quantities

$$
\begin{aligned}
\Lambda(\overline{M S}) r_{0} & =0.62(4)(4) ; \quad N_{f}=2 \\
& =0.60(8) . \quad N_{f}=0
\end{aligned}
$$

If we take $\Lambda(\overline{M S}) r_{0}=0.61(6)$ for $N_{f}=1$, then

$$
\frac{\Sigma}{\Lambda^{3}}=1.4(4)
$$

while ASV want 0.6 to 1.1 for the ratio. This is not so bad! (The difference between this and Eq. 1 is a 15 per cent shift between their $\Lambda$ and the interpolated ALPHA value.)

Armoni, Shore, and Veneziano have also predicted $\Sigma$ for other $N_{f}$ 's [37]. They add $N_{f}-1$ fundamental flavors to the mix, and get

$$
\frac{\Sigma}{\Lambda^{3}}=\frac{1}{4 \pi^{2}}\left(8-N_{f}\right)
$$

for the RGI condensate. They need a coupling constant to convert this to an $\overline{M S}$ number. Their publication only presents a band, since when they do the conversion from RGI to $\overline{M S}$ they consider a range of coupling constants. However, taking the $N_{f}=2$ value of $\Lambda(\overline{M S})$ from Eq. 10 and 
inverting it to give a coupling constant, $\Sigma^{1 / 3}=247 \mathrm{MeV}$. More accurate lattice measurements of $\Sigma$ (and $\Lambda$ ) vs $N_{f}$ could test the $N_{f}$ dependence in Eq. 12.

I am not sure what to do next. Unfortunately, the only nonperturbative quantity which can be computed in SYM is the gluino condensate (as far as I can tell, from asking many people), although to compensate, it is supposedly exact. So other predictions typically involve ratios of masses without some connection to the condensate:

$$
\frac{m_{\eta^{\prime}}^{2}}{m_{\sigma}^{2}}=1+O\left(1 / N_{c}\right)
$$

(From Ref. [2]; the $O\left(1 / N_{c}\right)$ corrections have been computed by Sannino and Shifman [38]), and a similar degeneracy for hybrids [39]. An $N_{f}=1$ meson is just like a flavor singlet meson in ordinary multiflavor QCD, so both the $\eta^{\prime}$ and $\sigma$ have disconnected ("hairpin") contributions. These are diffi cult and noisy. In addition, the scalar operator has a vacuum expectation value, so its signal is like a scalar glueball's: the exponential which gives the mass dives under a constant background. I have successfully avoided trying to do these for four months, now.

$N_{f}=1$ QCD has other intriguing properties: At negative quark mass, $N_{f}=1$ QCD may have a phase in which $\mathrm{CP}$ is broken. (This can occur for any $N_{f}$ if the quark mass matrix has positive and negative eigenvalues. This observation goes back to Dashen [40]; see Smilga [41] and Creutz [42] for recent discussions.) It is possible to simulate QCD at $m_{q}<0$ (or even complex mass) with overlap fermions by reweighting a real-mass simulation. The relevant derivation has been given by Dürr and Hoelbling [43], who briefly studied the Schwinger model. Complex mass is equivalent to a theta vacuum, another unvisited area of QCD for lattice simulators.

"The purpose of computing is insight, not numbers" (Hamming), so did we learn anything? It is always easiest to say No. The only quantitative prediction from $\mathscr{N}=1 \mathrm{SYM}$ is the condensate, and it has unknown $1 / N_{c}$ corrections. The spectrum of QCD depends on $N_{f}$ in an uncontrolled way. The only way to make predictions relevant to the real world is with simulations with three flavors of light quarks, all in the chiral regime. Even then, one must simulate at physical quark masses, unless the observable being studied has a well-behaved expansion in chiral perturbation theory allowing one to extrapolate in quark masses.

And yet-

When we teach about the spectrum of hydrogen in an introductory quantum mechanics class, the story is not "we do the calculation and the answer is $13.6 \mathrm{eV}$." There is systematic expansion (in $\alpha$ or equivalently in $v / c$ ) which allow us to make successively more accurate predictions. The parameters we have at our disposal in QCD are $N_{c}, N_{f}$, the color representations of the quarks, and the quark masses. Perhaps the zeroth order QCD calculation (like $V(r)=-e^{2} / r$ for hydrogen) is some extreme value of one or all of these parameters. Lattice tests of QCD-like theories might tell us new things about QCD, if they could validate extrapolations of analytic results from those theories. We won't know if we don't try!

And this test worked: ASV successfully predicted the $N_{f}=1$ condensate.

I would like to thank my collaborators Roland Hoffmann, Zhaofeng Liu, and Stefan Schaefer for many conversations, and I am grateful to Adi Armoni, Francesco Knechtli, Francesco Sannino, Misha Shifman, Matt Strassler, Mithat Ünsal, Gabriele Veneziano, and Larry Yaffe for correspon- 
dence. And thanks to the organizers for putting on a fantastic conference! This work was supported by the US Department of Energy.

\section{References}

[1] M. J. Strassler, arXiv:hep-th/0104032.

[2] A. Armoni, M. Shifman and G. Veneziano, Phys. Rev. Lett. 91, 191601 (2003) [arXiv:hep-th/0307097].

[3] A. Armoni, M. Shifman and G. Veneziano, Nucl. Phys. B 667, 170 (2003) [arXiv:hep-th/0302163].

[4] A. Armoni, M. Shifman and G. Veneziano, Phys. Lett. B 579, 384 (2004) [arXiv:hep-th/0309013].

[5] A. Armoni, M. Shifman and G. Veneziano, Phys. Rev. D 71, 045015 (2005) [arXiv:hep-th/0412203].

[6] G. 't Hooft, Nucl. Phys. B 75, 461 (1974).

[7] A. Armoni, M. Shifman and G. Veneziano, arXiv:hep-th/0403071.

[8] A. Patella, Phys. Rev. D 74, 034506 (2006) [arXiv:hep-lat/0511037].

[9] M. Unsal and L. G. Yaffe, arXiv:hep-th/0608180.

[10] N. M. Davies, T. J. Hollowood, V. V. Khoze and M. P. Mattis, Nucl. Phys. B 559, 123 (1999) [arXiv:hep-th/9905015].

[11] T. DeGrand and S. Schaefer, JHEP 0607, 020 (2006) [arXiv:hep-lat/0604015].

[12] T. DeGrand, R. Hoffmann, S. Schaefer and Z. Liu, Phys. Rev. D 74, 054501 (2006) [arXiv:hep-th/0605147].

[13] H. Leutwyler and A. Smilga, Phys. Rev. D 46, 5607 (1992).

[14] E. V. Shuryak and J. J. M. Verbaarschot, Nucl. Phys. A 560, 306 (1993) [arXiv:hep-th/9212088].

[15] J. J. M. Verbaarschot and I. Zahed, Phys. Rev. Lett. 70, 3852 (1993) [arXiv:hep-th/9303012].

[16] J. J. M. Verbaarschot, Phys. Rev. Lett. 72, 2531 (1994) [arXiv:hep-th/9401059].

[17] P. H. Damgaard, U. M. Heller, R. Niclasen and K. Rummukainen, Phys. Lett. B 495, 263 (2000) [arXiv:hep-lat/0007041].

[18] P. H. Damgaard and S. M. Nishigaki, Phys. Rev. D 63, 045012 (2001) [arXiv:hep-th/0006111].

[19] R. Sommer, Nucl. Phys. B 411, 839 (1994) [arXiv:hep-lat/9310022].

[20] G. Martinelli, C. Pittori, C. T. Sachrajda, M. Testa and A. Vladikas, Nucl. Phys. B 445, 81 (1995) [arXiv:hep-lat/9411010].

[21] H. Neuberger, Phys. Lett. B 417, 141 (1998) [arXiv:hep-lat/9707022].

[22] H. Neuberger, Phys. Rev. Lett. 81, 4060 (1998) [arXiv:hep-lat/9806025].

[23] P. H. Ginsparg and K. G. Wilson, Phys. Rev. D 25, 2649 (1982).

[24] A. Bode, U. M. Heller, R. G. Edwards and R. Narayanan, arXiv:hep-lat/9912043.

[25] N. Cundy, Nucl. Phys. Proc. Suppl. 153, 54 (2006) [arXiv:hep-lat/0511047].

[26] Z. Fodor, S. D. Katz and K. K. Szabo, JHEP 0408, 003 (2004) [arXiv:hep-lat/0311010].

[27] T. A. DeGrand and S. Schaefer, Phys. Rev. D 71, 034507 (2005) [arXiv:hep-lat/0412005]. 
[28] T. A. DeGrand and S. Schaefer, Phys. Rev. D 72, 054503 (2005) [arXiv:hep-lat/0506021].

[29] C. Morningstar and M. J. Peardon, Phys. Rev. D 69, 054501 (2004) [arXiv:hep-lat/0311018].

[30] T. A. DeGrand [MILC collaboration], Phys. Rev. D 63, 034503 (2001) [arXiv:hep-lat/0007046].

[31] William H. Press, Saul A. Teukolsky, William T. Vetterling, Brian P. Flannery, "Numerical Recipes in C++ : The Art of Scientific Computing", Cambridge Univ. Press, 2002.

[32] C. McNeile, Phys. Lett. B 619, 124 (2005) [arXiv:hep-lat/0504006].

[33] T. DeGrand, Z. Liu and S. Schaefer, arXiv:hep-lat/0608019.

[34] J. Gasser and H. Leutwyler, Phys. Lett. B 184, 83 (1987).

[35] S. Capitani, M. Luscher, R. Sommer and H. Wittig [ALPHA Collaboration], Nucl. Phys. B 544, 669 (1999) [arXiv:hep-lat/9810063].

[36] M. Della Morte, R. Frezzotti, J. Heitger, J. Rolf, R. Sommer and U. Wolff [ALPHA Collaboration], Nucl. Phys. B 713, 378 (2005) [arXiv:hep-lat/0411025].

[37] A. Armoni, G. Shore and G. Veneziano, Nucl. Phys. B 740, 23 (2006) [arXiv:hep-ph/0511143].

[38] F. Sannino and M. Shifman, Phys. Rev. D 69, 125004 (2004) [arXiv:hep-th/0309252].

[39] A. Gorsky and M. Shifman, Phys. Rev. D 71, 025009 (2005) [arXiv:hep-th/0410099].

[40] R. F. Dashen, Phys. Rev. D 3, 1879 (1971).

[41] A. V. Smilga, Phys. Rev. D 59, 114021 (1999) [arXiv:hep-ph/9805214].

[42] M. Creutz, Rev. Mod. Phys. 73, 119 (2001) [arXiv:hep-lat/0007032].

[43] S. Durr and C. Hoelbling, Phys. Rev. D 74, 014513 (2006) [arXiv:hep-lat/0604005]. 